\title{
An improved accurate monotonicity-preserving scheme for the Euler equations
}

\author{
Zhiwei Hea ${ }^{\mathrm{a}}$, Yousheng Zhanga ${ }^{\mathrm{a}}$, Fujie Gao ${ }^{\mathrm{a}}$, Xinliang $\mathrm{Li}^{\mathrm{b}}$, Baolin Tian ${ }^{\mathrm{a}, *}$ \\ a Laboratory of Computational Physics, Institute of Applied Physics and Computational Mathematics, Beijing 100094, China \\ ${ }^{\mathrm{b}}$ State Key Laboratory of High-temperature Gas Dynamics, Institute of Mechanics, Chinese Academy of Sciences, Beijing 100190, China
}

\section{A R T I C L E I N F O}

\section{Article history:}

Received 21 October 2015

Revised 28 July 2016

Accepted 8 September 2016

Available online 10 September 2016

\section{MSC:}

00-01

99-00

\section{Keywords:}

Monotonicity-preserving

Accuracy-preserving

TVD

Flux limiter

Hyperbolic conservation laws

\begin{abstract}
A B S T R A C T
The accurate monotonicity-preserving (MP) scheme of Suresh and Huynh (1997) [5] is a high-order and high-resolution method for hyperbolic conservation laws. However, the robustness of the MP scheme is not very high. In this paper, a detailed analysis on this scheme is performed, and two potential causes which may account for the weak robustness are revealed. Furthermore, in order to enhance the robustness of the MP scheme, an improved version of the MP scheme is presented, in which a strict continuous total-variation-diminishing (TVD) numerical flux is used at a disturbed discontinuity so that oscillations cannot grow indefinitely without violating the TVD condition. Without destroying the very high resolution property, numerical tests show that the improved scheme shares a strong robustness in simulating extreme numerical tests.
\end{abstract}

(c) 2016 Elsevier Ltd. All rights reserved.

\section{Introduction}

As is well known, flows involving multi-scales and discontinuities occur widely in many natural phenomena and engineering applications [1,2]. In terms of simulating the flows, numerical schemes must be high-order accurate in smooth regions to resolve the multi-scales, and essentially oscillation-free near discontinuities to capture features such as shock waves. Consequently, the development of high-order shock-capturing schemes is of importance.

Van Leer [3] first showed that it is beneficial to strive for schemes with a high order of accuracy while capturing shock waves in an essentially oscillation-free manner. By using piecewise linear interpolation, coupled with a limiting strategy to control oscillations at discontinuities, he designed a second-order monotonicity-preserving version of the Godunov scheme. Later, Colella and Woodward [4] developed the piecewise parabolic method (PPM), which employs a four-point centered stencil to define the numerical flux; this numerical flux is then limited to control oscillations. Following this limiting approach, Suresh and Huynh [5] proposed the accurate monotonicity-preserving (MP)

\footnotetext{
* Corresponding author.

E-mail address: tian_baolin@iapcm.ac.cn (B. Tian).
}

scheme. In the MP scheme, starting with a primary numerical flux calculated by any high-order scheme, the following two procedures are executed: (1) calculate a local interval, which is designed by enlarging the first-order monotonicity-preserving interval derived in [5], and (2) maintain/replace the primary numerical flux according to the relation between the primary numerical flux and this local interval: (i) if the primary numerical flux lies in this local interval, it is maintained, and (ii) if the primary numerical flux is beyond this local interval, it is replaced by the nearest bound of this local interval. The key feature of the MP scheme is that this local interval is designed to have the following property-it is very large in smooth regions so that it contains the primary numerical flux, and automatically degenerates to the monotonicitypreserving interval at discontinuities. That is to say, this local interval will enlarge for non-monotonic data in order to achieve the accuracy-preserving property, and automatically degenerates to the monotonicity-preserving interval for monotonic data. Due to this property, the total variation of the numerical solution in the MP scheme is allowed to increase only for non-monotonic data, and diminish for monotonic data [6].

The recent development of the MP scheme mainly focuses on the problem of calculating the primary numerical flux. In the original paper, the fifth-order upwind scheme is adopted [5]. Later, some other schemes are tested, such as the fifth-order compact upwind scheme [7] or center schemes with controllable 
artificial dissipation [8,9]. Balsara and Shu [10] even adopt a highorder weighted essentially non-oscillatory (WENO) scheme to obtain the primary numerical flux, yielding high-order monotonicitypreserving WENO schemes. Daru and Tenaud [6] reinterpreted the local interval in MP schemes as TVD-like conditions, and applied these conditions to a one-step scheme.

The MP scheme shows high-resolution for multi-scale problems. Therefore, it is widely adopted in practical applications [11,12]. However, the robustness of the MP scheme is not very high, compared with the popular WENO methods [13]. In this paper, we perform a detailed analysis of the MP scheme, trying to reveal the potential causes which may account for the weak robustness of the MP scheme. Furthermore, in order to enhance the robustness of the MP scheme, a simple modification is proposed to suppress the numerical oscillations more efficiently and/or to prevent the appearance of numerical oscillations as possible. Numerical tests show that the improved MP scheme shows a more strong robustness.

\section{Method}

\subsection{Framework}

Consider the following one-dimensional linear advection equation

$\frac{\partial u}{\partial t}+\frac{\partial f(u)}{\partial x}=0$

with constant advection speed, i.e. $\frac{d f}{d u}=a$. Without loss of generality, assume that $a \geq 0$. Eq. (1) is discretized in a uniform grid defined by the points $x_{i}=i \Delta x, i=1, \cdots, N$, with cell boundaries given by $x_{i+1 / 2}=x_{i}+\frac{\Delta x}{2}$, where $\Delta x$ is the uniform grid spacing. The spatial discretization is obtained by implicitly defining the numerical flux function $h(x)$ as

$f(x)=\frac{1}{\Delta x} \int_{x-\frac{\Delta x}{2}}^{x+\frac{\Delta x}{2}} h(\xi) \mathrm{d} \xi$,

such that the spatial derivative in Eq. (1) is exactly approximated by a conservative finite difference formula at the cell boundaries,

$\frac{\mathrm{d} u_{i}(t)}{\mathrm{d} t}=-\frac{h_{i+1 / 2}-h_{i-1 / 2}}{\Delta x}$,

where $h_{i \pm \frac{1}{2}}=h\left(x_{i \pm \frac{1}{2}}\right)$, and $u_{i}(t)$ is a numerical approximation to the point value $u\left(x_{i}, t\right)$. In practice, Eq. (3) is approximated as

$\frac{\mathrm{d} u_{i}(t)}{\mathrm{d} t} \approx-\frac{\widehat{f}_{i+1 / 2}-\widehat{f}_{i-1 / 2}}{\Delta x}$,

where numerical fluxes $\widehat{f}_{i \pm \frac{1}{2}}$, reconstructed from known cell average values $f_{i}$, are approximations of $h_{i \pm \frac{1}{2}}$. Then Eq. (4) can be marched by one time step or substep of TVD Runge-Kutta schemes [14]:

$u_{i}^{n+1} \approx u_{i}^{n}-\frac{\Delta t}{\Delta x}\left(\widehat{f}_{i+1 / 2}^{n}-\widehat{f}_{i-1 / 2}^{n}\right)$,

where $\Delta t$ is the time step, $u_{i}^{n+1}$ the numerical approximation to the point value $u\left(x_{i}, t\right)$ at time level $t^{n+1}$, and $\widehat{f}_{i \pm \frac{1}{2}}^{n}$ is the numerical flux at time level $t^{n}$ (the superscript $n$ in $\widehat{f}_{i \pm \frac{1}{2}}^{n}$ is omitted for brevity hereafter).

\subsection{The accurate monotonicity-preserving (MP) scheme}

When the MP scheme [5] is used to calculate the numerical flux $\widehat{f}_{i+1 / 2}$ in Eq. (5), it is implemented as follows.

\subsubsection{Some notations used in the MP scheme}

First of all, we introduce some definitions used in the accurate monotonicity-preserving (MP) scheme. The first one is the minmod function for $q$ arguments [15]:

$\operatorname{minmod}\left(z_{1}, z_{2}, \cdots z_{q}\right):=s \cdot \min \left(\left|z_{1}\right|,\left|z_{2}\right|, \cdots,\left|z_{q}\right|\right)$,

where

$$
\begin{aligned}
s= & \frac{1}{2}\left(\operatorname{sgn}\left(z_{1}\right)+\operatorname{sgn}\left(z_{2}\right)\right) \mid \frac{1}{2}\left(\operatorname{sgn}\left(z_{1}\right)\right. \\
& \left.+\operatorname{sgn}\left(z_{3}\right)\right) \cdots \frac{1}{2}\left(\operatorname{sgn}\left(z_{1}\right)+\operatorname{sgn}\left(z_{q}\right)\right) \mid,
\end{aligned}
$$

and $\operatorname{sgn}(z)$ is a function that returns the sign of the argument $z$. The second one is the definition of an interval [15]:

$I\left[z_{1}, z_{2}, \cdots, z_{q}\right]:=\left[\min \left(z_{1}, z_{2}, \cdots, z_{q}\right), \max \left(z_{1}, z_{2}, \cdots, z_{q}\right)\right]$.

The third one is the definition of the local curvature $d_{i+1 / 2}^{M M}[5]$ :

$d_{i+1 / 2}^{M M}:=\operatorname{minmod}\left(d_{i}, d_{i+1}\right)$

where $d_{i}=f_{i+1}+f_{i-1}-2 f_{i}$.

\subsubsection{Review of the MP scheme}

First, obtain the primary numerical flux $\widehat{f}_{i+1 / 2}^{o}$ using a highorder scheme. In the original paper of the MP scheme [5], the following fifth-order upwind scheme (U5)

$\widehat{f}_{i+1 / 2}^{o}=\frac{2 f_{i-2}-13 f_{i-1}+47 f_{i}+27 f_{i+1}-3 f_{i+2}}{60}$

is used. Then, the primary numerical flux $\widehat{f}_{i+1 / 2}^{o}$ is maintained or replaced according to the following limiting procedures.

First, in order to achieve the monotonicity-preserving property: (I) $\widehat{f}_{i+1 / 2}^{o}$ should lie between $f_{i}$ and $f_{i+1}$. (II) $u_{i}^{n+1}$ should lie between $u_{i-1}$ and $u_{i}$, which merely ensures that $\widehat{f}_{i+1 / 2}^{o}$ lies between $f_{i}$ and $f^{U L}$, where

$f^{U L}=f_{i}+\kappa\left(f_{i}-f_{i-1}\right)$,

and $\kappa \geq 2$ [5]. Combining the two assumptions, a first-order monotonicity-preserving interval $I\left[f_{i}, f^{\mathrm{MP}}\right]$, which is just the intersection of $I\left[f_{i}, f_{i+1}\right]$ and $I\left[f_{i}, f^{U L}\right]$, is derived, where

$f^{M P}=f_{i}+\operatorname{minmod}\left(f_{i+1}-f_{i}, \kappa\left(f_{i}-f_{i-1}\right)\right)$.

However, to bring $\widehat{f}_{i+1 / 2}^{O}$ into the interval $I\left[f_{i}, f^{M P}\right]$ will result in degeneration to first order near an extremum. In order to avoid this drawback, Suresh and Huynh [5] proposed the idea of enlarging the intervals defined above to avoid the loss of accuracy. Based on the parabolic interpolation, $I\left[f_{i}, f_{i+1}\right]$ and $I\left[f_{i}, f^{U L}\right]$ were enlarged to $I\left[f_{i}, f_{i+1}, f^{M D}\right]$ and $I\left[f_{i}, f^{U L}, f^{L C}\right]$, respectively, where

$f^{M D}=\frac{1}{2}\left(f_{i}+f_{i+1}\right)-\frac{1}{2} d_{i+1 / 2}^{M M}$,

$f^{L C}=f_{i}+\frac{1}{2}\left(f_{i}-f_{i-1}\right)+\frac{4}{3} d_{i-1 / 2}^{M M}$,

or [6]

$f^{L C}=\frac{1}{2}\left(f_{i}+f^{U L}\right)+\frac{\kappa}{2} d_{i-1 / 2}^{M M}$.

Moreover, it has been proven that $I\left[f_{i}, f_{i+1}, f^{M D}\right]$ and $I\left[f_{i}, f^{U L}, f^{L C}\right]$ will enlarge only for non-monotonic numerical data, and will automatically degenerate to $I\left[f_{i}, f_{i+1}\right]$ and $I\left[f_{i}, f^{U L}\right]$ for monotonic numerical data $[5,6]$. However, in practice it is recommended to replace $d_{i+1 / 2}^{M M}$ with a more restrictive but heuristic measure of the local curvature $d_{i+1 / 2}^{M 4}$ [5]

$d_{i+1 / 2}^{M 4}=\operatorname{minmod}\left(d_{i}, d_{i+1}, 4 d_{i}-d_{i+1}, 4 d_{i+1}-d_{i}\right)$. 
Finally, the local interval $I\left[f^{\min }, f^{\max }\right]$, which is just the intersection of $I\left[f_{i}, f_{i+1}, f^{M D}\right]$ and $I\left[f_{i}, f^{U L}, f^{L C}\right]$, is obtained, where

$f^{\min }=\max \left(\min \left(f_{i}, f_{i+1}, f^{M D}\right), \min \left(f_{i}, f^{U L}, f^{L C}\right)\right)$,

$f^{\max }=\min \left(\max \left(f_{i}, f_{i+1}, f^{M D}\right), \max \left(f_{i}, f^{U L}, f^{L C}\right)\right)$.

If the primary numerical flux is outside this interval, it is replaced by the nearest bound of the interval. That is, the accurate monotonicity-preserving (MP) schemes reads as

$\widehat{f}_{i+1 / 2}=\widehat{f}_{i+1 / 2}^{o}+\operatorname{minmod}\left(f^{\max }-\widehat{f}_{i+1 / 2}^{o}, f^{\min }-\widehat{f}_{i+1 / 2}^{o}\right)$.

\section{Comments on the accurate monotonicity-preserving scheme}

In practice, we often use the MP scheme to solve nonlinear problems. However, compared with the popular WENO methods [13], the robustness of the MP scheme is not very high. In this section, we try to investigate the potential causes.

\subsection{Comment I: suppress numerical oscillations at disturbed discontinuities}

When solving nonlinear problems, we encounter various numerical oscillations, and some of which are unavoidable [16]. At discontinuities, there are, say, overshoot/undershoot oscillations (Gibbs phenomenon) and post-shock oscillations [16]. These oscillations (spurious waves) have non-monotonic appearances in physical space. Therefore, $I\left[f_{i}, f_{i+1}, f^{M D}\right]$ and $I\left[f_{i}, f^{U L}, f^{L C}\right]$ will become larger.

To illustrate this condition more clearly, consider the following numerical data $u\left(x ; x_{0}\right)=u_{0}\left(x ; x_{0}\right)+u_{d i s}\left(x ; x_{0}\right)$, in which the disturbance $u_{\text {dis }}\left(x ; x_{0}\right)$ is defined as

$u_{d i s}\left(x ; x_{0}\right)=A \cdot \sin \left(\frac{2 \pi\left(x-x_{0}\right)}{b_{1} \Delta x}\right) \cdot \exp \left(-\ln (2)\left(\frac{x-x_{0}}{b_{2} \Delta x}\right)^{2}\right)$,

where $b_{1} \Delta x$ is the dominant wavelength and $b_{2} \Delta x$ the half-width of the Gaussian function. The parameters $A, b_{1}$ and $b_{2}$ are directly connected to the spectral contents of the disturbance [17]. For this case, we set $x_{0}=0, A=0.2, b_{1}=8$ and $b_{2}=3$. This disturbance is characterized by wavenumbers in the range $0<k \Delta x<\pi / 2$ with a peak for $k \Delta x=\pi / 4$, i.e., for eight points per wavelength [17]. Furthermore, in order to model a discontinuity, we set $u_{0}$ as

$u_{0}\left(x ; x_{0}\right)=\tanh \left(\sigma\left(x-x_{0}\right)\right)$

with $\sigma=50$. Fig. 1 (a) shows the profiles of $u\left(x ; x_{0}\right)$ over the $[-1,1]$ domain with 101 grid points. From Fig. 1(a), we can see that the monotonicity of the discontinuity $u_{0}$ is destroyed due to the appearance of the disturbance $u_{\text {dis }}$.

In order to detect whether $I\left[f^{\min }, f^{\max }\right]$ enlarges, we define an indicator $\Theta$ at $i+1 / 2$ as follows:

$\Theta= \begin{cases}1, & \text { if }\left(f^{\max }-f^{\min }\right)>\left(f_{0}^{\max }-f_{0}^{\min }\right) \\ 0, & \text { else }\end{cases}$

where $f_{0}^{\min }=\min \left(f_{i}, f^{M P}\right)$ and $f_{0}^{\max }=\max \left(f_{i}, f^{M P}\right)$. If $I\left[f^{\min }, f^{\max }\right]$ becomes larger, $\Theta=1$; otherwise, $\Theta=0$. Fig. 1(b) shows the profiles of $\Theta$ at cell boundaries $x_{i+1 / 2}$ within the domain [-0.4, 0.4]. From Fig. 1(b), we can clearly see that the two intervals $I\left[f_{i}, f_{i+1}, f^{M D}\right]$ and $I\left[f_{i}, f^{U L}, f^{L C}\right]$ do become larger due to the appearance of the disturbance.

The final step in the MP scheme (Eq. (17)) means that if the primary numerical flux $\widehat{f}_{i+1 / 2}^{o}$ does not lie in $I\left[f^{\min }, f^{\max }\right]$, it will be replaced by $f^{\min }$ or $f^{\max }$, depending on which is closer to $\widehat{f}_{i+1 / 2}^{o}$. That is to say, the MP scheme suppresses these numerical oscillations by using two sub-scheme: $f^{\min }$ or $f^{\max }$.
Therefore, due to the enlargement of $I\left[f^{\mathrm{min}}, f^{\max }\right]$, the MP scheme will provide much room for the growth of "oscillations" when the discontinuity is disturbed. However, this is not a desired property when we solve nonlinear problems; oscillations at discontinuities are best to be damped for sake of robustness of the code.

\subsection{Comment II: numerical oscillations introduced by hybridization}

Furthermore, when the primary numerical flux $\widehat{f}_{i+1 / 2}^{o}$ is replaced by $f^{\min }$ (for the $f^{\max }$, the condition is the same), the value of $f^{\text {min }}$ is abruptly chosen according to one of five sub-schemes: $f_{i}$, $f_{i+1}, f^{M D}$, f $f^{U L}$, and $f^{L C}$. That is to say, the final numerical flux $\widehat{f}_{i+1 / 2}$ may become a hybrid scheme between $f_{i}, f_{i+1}, f^{M D}, f^{U L}$, and $f^{L C}$. However, it has already been found that, in hybrid schemes, some spurious waves may be generated at interfaces where one subscheme is switched abruptly to another sub-scheme [18]. These spurious waves could eventually propagate into the smooth regions [18].

Remark 1. In fact, if the high-order method used in the MP scheme to obtain $\widehat{f}_{i+1 / 2}^{o}$ does not have sufficient dissipation, parasitic physical solutions may be obtained. In smooth regions, it is well known that any finite difference scheme on a specific grid has a range of wavenumbers that can be well resolved. Waves with higher wavenumbers that are beyond this range will disperse, causing dispersive errors [19]. Furthermore, the energy of waves of higher wavenumbers that cannot be represented on the grid is aliased onto the resolved wavenumbers, resulting in aliasing errors [20]. Because the primary numerical flux is maintained in smooth regions, these spurious waves will continually parasitize the physical solutions.

\section{An improved accurate monotonicity-preserving scheme}

In the last section, we have tried to investigate the potential causes for the weak robustness of the MP scheme. In this section, we will give specific strategies to suppress the numerical oscillations more efficiently and/or to prevent the appearance of numerical oscillations as possible.

\subsection{A simple modification}

Note that oscillations always add to the total variation. Therefore, oscillations cannot grow indefinitely without violating the TVD condition. That is to say, if we enforce the TVD constraint, the size of the oscillations must eventually decrease.

When $I\left[f^{\min }, f^{\max }\right]$ becomes larger at a disturbed discontinuity, we use the following TVD numerical flux on the stencil $\left[x_{i-1}, x_{i}, x_{i+1}\right]$,

$\widehat{f}_{i+1 / 2}=f_{i}+\frac{1}{\phi_{c}} \phi_{i+1 / 2}\left(r_{i+1 / 2}\right)\left(f_{i+1}-f_{i}\right)$,

where $r_{i+1 / 2}=\frac{f_{i}-f_{i-1}}{f_{i+1}-f_{i}}$, the flux limiter $\phi_{i+1 / 2}\left(r_{i+1 / 2}\right)$ is a function of $r_{i+1 / 2}$, and $\phi_{c}$ is a positive constant, typically 2 [21]. Furthermore, the flux limiter $\phi_{i+1 / 2}\left(r_{i+1 / 2}\right)$ satisfies the classical TVD constraints [22]

$0 \leq \phi_{i+1 / 2} \leq \phi_{c}$

$0 \leq \phi_{i+1 / 2} \leq \phi_{c} r_{i+1 / 2} \frac{1-v}{v}$.

where $v=a \frac{\Delta t}{\Delta x}$ is the CFL number. That is to say, Eq. (21) with any flux limiter that satisfies the constraints (Eqs. (22) and (23)) is a TVD numerical flux. 

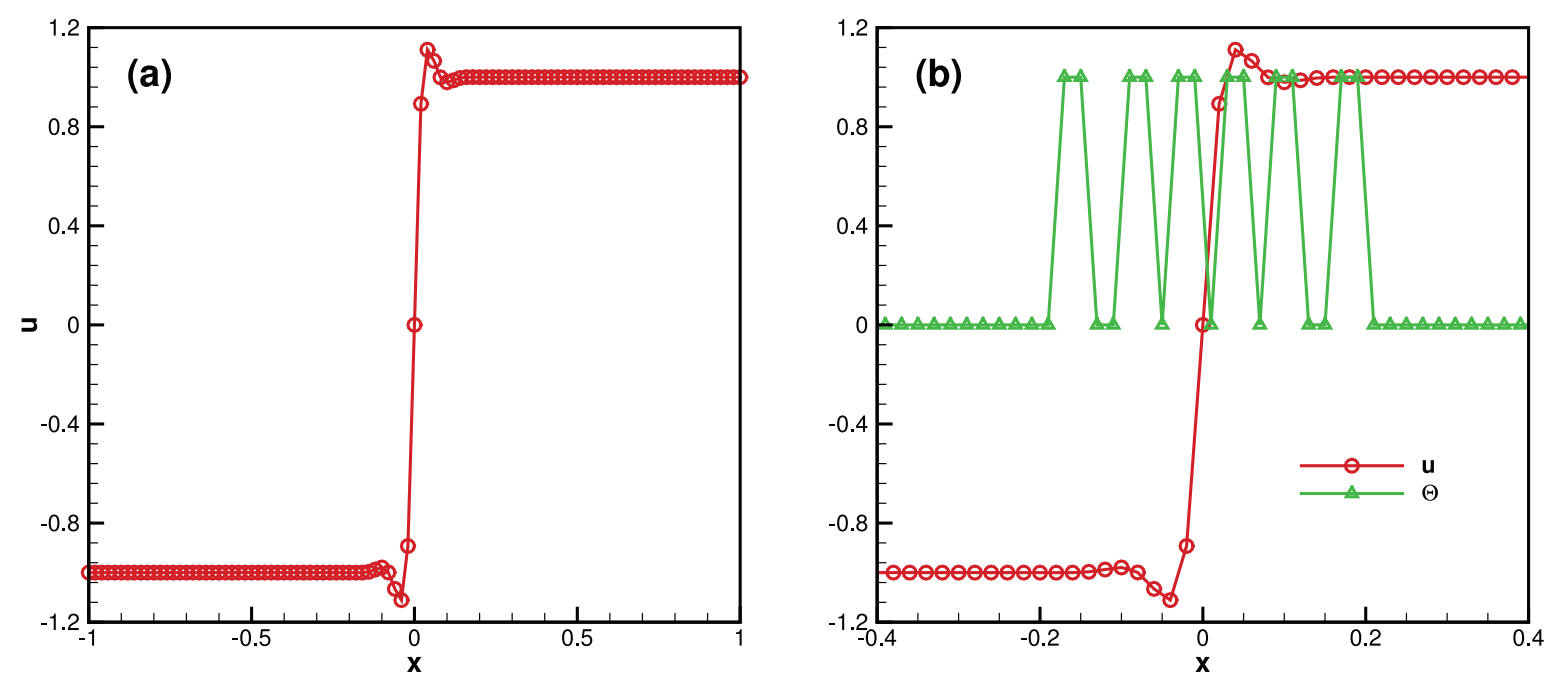

Fig. 1. Profiles of: (a) $u\left(x ; x_{0}\right)$ over the $[-1,1]$ domain with 101 grid points; (b) $\Theta$ at cell boundaries $x_{i+1 / 2}$ within the domain [-0.4, 0.4$]$.

The logic behind the introduction of Eq. (21) is as follows. First, it has shown that Eq. (21) with the constraints (Eqs. (22) and (23)) is identical to the monotonicity-preserving interval derived in MP schemes [6]. Therefore, the use of with any limiter (satisfies Eq. (22) and Eq. (23)) meets the monotonicity-preserving interval derived in the MP scheme. Second, it is well known that the flux limiter is usually set to zero for negative values of $r$. Therefore, the TVD numerical flux (Eq. (21)) will reduce to the first-order monotonic scheme. Due to this property, the oscillation (which exhibits itself as local extrema) at discontinuities will be damped.

Furthermore, in calculating the TVD numerical flux (Eq. (21)), one needs a specific flux limiter. As discussed in Section 3.2, some spurious waves might be generated by the abrupt transition in a hybrid scheme. A continuous (smooth is better) transition from one sub-scheme to another is suggested [23]. To be specific, the hybrid scheme is suggested to be designed as some continuous weighted average of sub-schemes [23]. Since the aim of this paper is to suppress numerical oscillations more efficiently and/or to prevent the appearance of numerical oscillations as possible, we follow this suggestion to reduce numerical oscillations by hybridization as possible. Therefore, the van Leer limiter [24]:

$\phi_{i+1 / 2}\left(r_{i+1 / 2}\right)=\frac{r_{i+1 / 2}+\left|r_{i+1 / 2}\right|}{1+\left|r_{i+1 / 2}\right|}$,

is adopted, where $r_{i+1 / 2}=\frac{\Delta f_{i-1 / 2}}{\Delta f_{i+1 / 2}}, \quad \Delta f_{i-1 / 2}=f_{i}-f_{i-1} \quad$ and $\Delta f_{i+1 / 2}=f_{i+1}-f_{i}$.

\subsection{The improved version of the MP scheme}

The advantage of the interval $I\left[f^{\min }, f^{\max }\right]$ is that, it becomes larger near local extrema in a smooth region. Therefore, it provides room so that the primary numerical flux $\widehat{f}_{i+1 / 2}^{o}$ will lie in the interval and will be maintained. Thus the accuracy is preserved near local extrema in smooth regions.

Considering this property of $I\left[f^{\min }, f^{\max }\right]$ and the discussions in Section 4.1, we can propose an improved accurate MP scheme as follows. If the primary numerical flux $\widehat{f}_{i+1 / 2}^{o}$ does not lie in the interval $I\left[f^{\min }, f^{\max }\right]$, the TVD numerical flux (Eq. (21) with the limiter (24) is considered. If $\widehat{f}_{i+1 / 2}^{o}$ lies in $I\left[f^{\min }, f^{\max }\right]$, the accuracy should be preserved due to the use of a high-order scheme to obtain $\widehat{f}_{i+1 / 2}^{o}$. This mechanism can be achieved by the following spe- cific procedures

$$
\begin{aligned}
\widehat{f}_{i+1 / 2}= & \frac{\widehat{f}_{i+1 / 2}^{O}+\widehat{f}_{i+1 / 2}^{R e}}{2}-\operatorname{sgn}\left(\left(\widehat{f}_{i+1 / 2}^{O}-f^{\mathrm{min}}\right) \cdot\left(\widehat{f}_{i+1 / 2}^{O}-f^{\max }\right)\right) \\
& \times \frac{\widehat{f}_{i+1 / 2}^{o}-\widehat{f}_{i+1 / 2}^{R e}}{2}
\end{aligned}
$$

where

$$
\begin{aligned}
\widehat{f}_{i+1 / 2}^{R e}= & f_{i}+\frac{\operatorname{sgn}\left(\Delta f_{i-1 / 2}\right)+\operatorname{sgn}\left(\Delta f_{i+1 / 2}\right)}{2} \\
& \times \frac{\left|\Delta f_{i-1 / 2}\right|\left|\Delta f_{i+1 / 2}\right|}{\left|\Delta f_{i-1 / 2}\right|+\left|\Delta f_{i+1 / 2}\right|+\epsilon}
\end{aligned}
$$

and $\epsilon=10^{-40}$. The improved accurate monotonicity-preserving scheme is referred to as MP-R.

Remark 2. Dissipative schemes, which can damp various spurious waves in smooth regions, should be used. With this strategy, spurious waves are eliminated while accuracy is still high-order if the primary numerical flux is not further limited. However, a quantitative criterion to determine a sufficient dissipation has not been established so far [19], although there are some heuristic choices. Some researches use center schemes with artificially controllable dissipation $[8,9]$. However, it is not certain to what extent the dissipations of these schemes are proper. It is more common to use upwind schemes, such as the fifth-order upwind scheme (Eq. (9)) or the following fifth-order upwind compact scheme [7]

$\widehat{f}_{i+1 / 2}^{o}=\frac{-24 \widehat{f}_{i-1 / 2}+3 f_{i-1}+47 f_{i}+11 f_{i+1}-f_{i+2}}{36}$.

In this paper, we continue to use the fifth-order upwind scheme that was used in the original MP scheme.

\section{Numerical tests}

Several numerical tests in one- and two-dimensional spaces were performed. In the rest of this paper, MP5-R refers to the fifth-order improved MP scheme; MP5 refers to the fifth-order MP scheme [5]; "WENO5" refers to the fifth-order WENO scheme [13]. For the linear case, the governing equation is

$u_{t}+u_{x}=0$.

For 1D nonlinear cases, the governing equations are the 1D Euler equations:

$\mathbf{u}_{t}+[\mathbf{f}(\mathbf{u})]_{x}=0$, 
where $\mathbf{u}$ and $\mathbf{f}$ are given in the following form:

$\mathbf{u}=\left(\begin{array}{c}\rho \\ \rho u \\ \rho E\end{array}\right), \mathbf{f}=\left(\begin{array}{c}\rho u \\ \rho u^{2}+p \\ (\rho E+p) u\end{array}\right)$.

Here, $\rho$ is the density, $u$ is the velocity, $E=\rho e+\frac{u^{2}}{2}$ is the total energy, $e$ is the internal energy, and $p$ is the pressure. In this study, the ideal equation of state $p=(\gamma-1) \rho e$ is used, where $\gamma$ is the ratio of the specific heats. If not mentioned otherwise, $\gamma=1.4$. The 1D Euler equations are solved by following the general characteristic-wise methodology $[13,25]$. Specifically, the Roe approximation is used for the characteristic decomposition at the cell faces, and the Lax-Friedrichs flux splitting method [26,27] is used in each characteristic field. For two-dimensional cases, the 2D Euler equations are solved in a dimension-by-dimension fashion. A third-order TVD Runge-Kutta scheme [14] is used for the time integration. Because $\kappa$ in the left-sided upper limit $f^{U L}$ is CFL-dependent, and should be $\frac{1-v}{v}$, the value of $\kappa$ is set to 4 [5] which theoretically leads to a CFL number restriction $v \leq 0.2$ while $v=0.4$ still yields non-oscillatory results [5]. Therefore, the CFL number is set to 0.4 for all computations.

\subsection{Advection of an initial profile with discontinuities}

First of all, we consider the classical test case of the advection of an initial profile composed of a Gaussian wave, a square wave, a triangular wave and an ellipse [6]. This is a difficult test case because it includes discontinuities as well as smooth portions of curves and extrema [6]. Therefore, we use this case to show abilities of the MP5-R scheme of accuracy-preserving and suppressing the numerical oscillations more efficiently at discontinuities.

The initial condition is defined on the domain $[-1,1]$ as:

$$
u(x, 0)=u_{0}(x)
$$$$
= \begin{cases}\exp \left(-\log (2)(x+0.7)^{2} / 0.0009\right), & \text { if }-0.8 \leq x \leq-0.6 \\ 1, & \text { if }-0.4 \leq x \leq-0.2 \\ 1-|10(x-0.1)|, & \text { if } 0 \leq x \leq 0.2 \\ \sqrt{1-100(x-0.5)^{2},} & \text { if } 0 \leq x \leq 0.2 \\ 0, & \text { otherwise }\end{cases}
$$

and periodic boundary conditions are prescribed. As done in [6], we use uniform grid composed of 200 mesh-cells, and the solution obtained at $t=20$ (10 periods) are shown in Fig. 2.

From Fig. 2, it can be seen that the high-resolution property of the MP5-R scheme are essentially the same as that of the MP5 scheme. In order to show the ability of the MP5-R scheme of suppressing the numerical oscillations more efficiently at disturbed discontinuities, we add some disturbances (see Eq. (18)) to the initial conditions :

$u(x, 0)=u_{0}(x)+u_{d i s}(x ;-0.4)-u_{d i s}(x ;-0.2)$.

From Fig. 3, we can see that there are obvious oscillations at disturbed discontinuities. After this long time integration, the MP5 scheme still preserve the oscillations to some extent, which may seen clearly in Fig. 3. In contrast, the MP5-R scheme has already damped the oscillations at disturbed discontinuities. Therefore, we can claim that the MP5-R scheme will suppress numerical oscillations more efficiently than the MP5 scheme when we solve nonlinear problems.

\subsection{Resolution tests}

In this section, we use two typical tests: the shock density-wave interaction problem [26] and the double Mach reflection problem [28], to show the high resolution property of the new method.

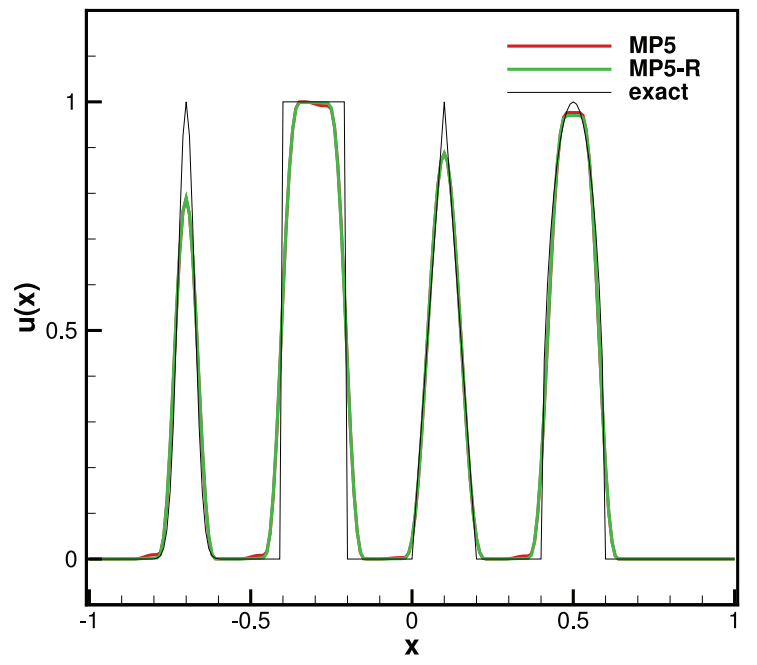

Fig. 2. Advection of an initial profile with discontinuities over 10 periods $(t=20)$ by MP5-R and MP5. (For interpretation of the references to color in this figure legend, the reader is referred to the web version of this article.)

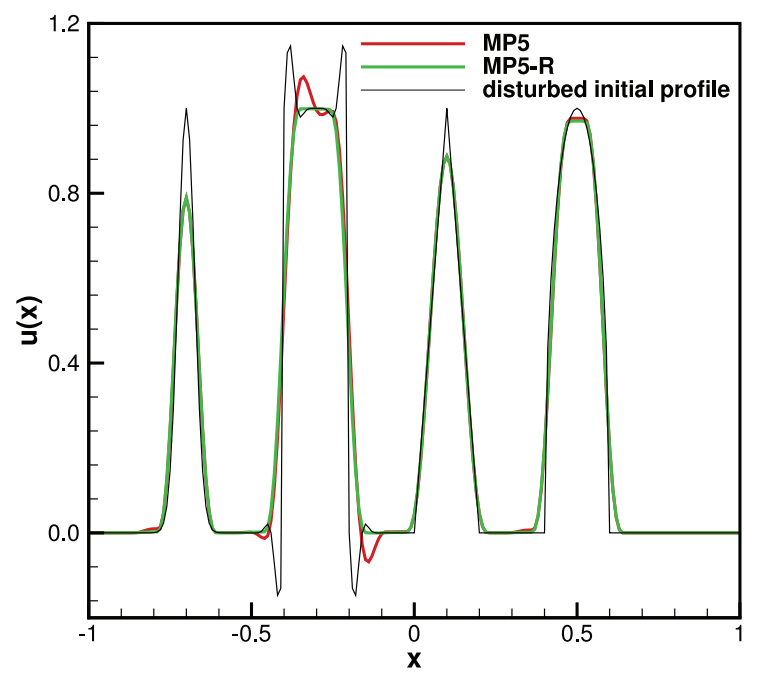

Fig. 3. Advection a disturbed initial profile over 10 periods $(t=20)$ by MP5-R and MP5. (For interpretation of the references to color in this figure legend, the reader is referred to the web version of this article.)

\subsubsection{The shock density-wave interaction problem}

The initial conditions are set by a Mach 3 shock interacting with a perturbed density field

$$
(\rho, u, p)= \begin{cases}(3.857,2.629,10.333), & \text { if } 0 \leq x \leq 1 \\ (1+0.2 \sin (5 x), 0,1), & \text { if } 1 \leq x \leq 10\end{cases}
$$

and the final time is $t=1.8$. A zero-gradient boundary condition is applied at $x=0$ and $x=10$. Moreover, we shall refer to the solution computed by the classical WENO5 scheme with 5001 grid points as the "reference" solution.

Fig. 4 shows the calculated density profiles at $t=1.8$ on a grid with 201 grid points. From Fig. 4, we can see that a good agreement with the reference solution is obtained for all schemes. However, the MP5-R scheme captures much more fine scale structures of the solution than WENO5, particularly in the high-frequency waves behind the shock. The results obtained by the MP5 scheme are also plotted in Fig. 4. It can be seen that the high-resolution property of the MP5-R scheme is essentially the same as those of the MP5 scheme. More importantly, we will show that the MP5-R scheme further shows high robustness. 

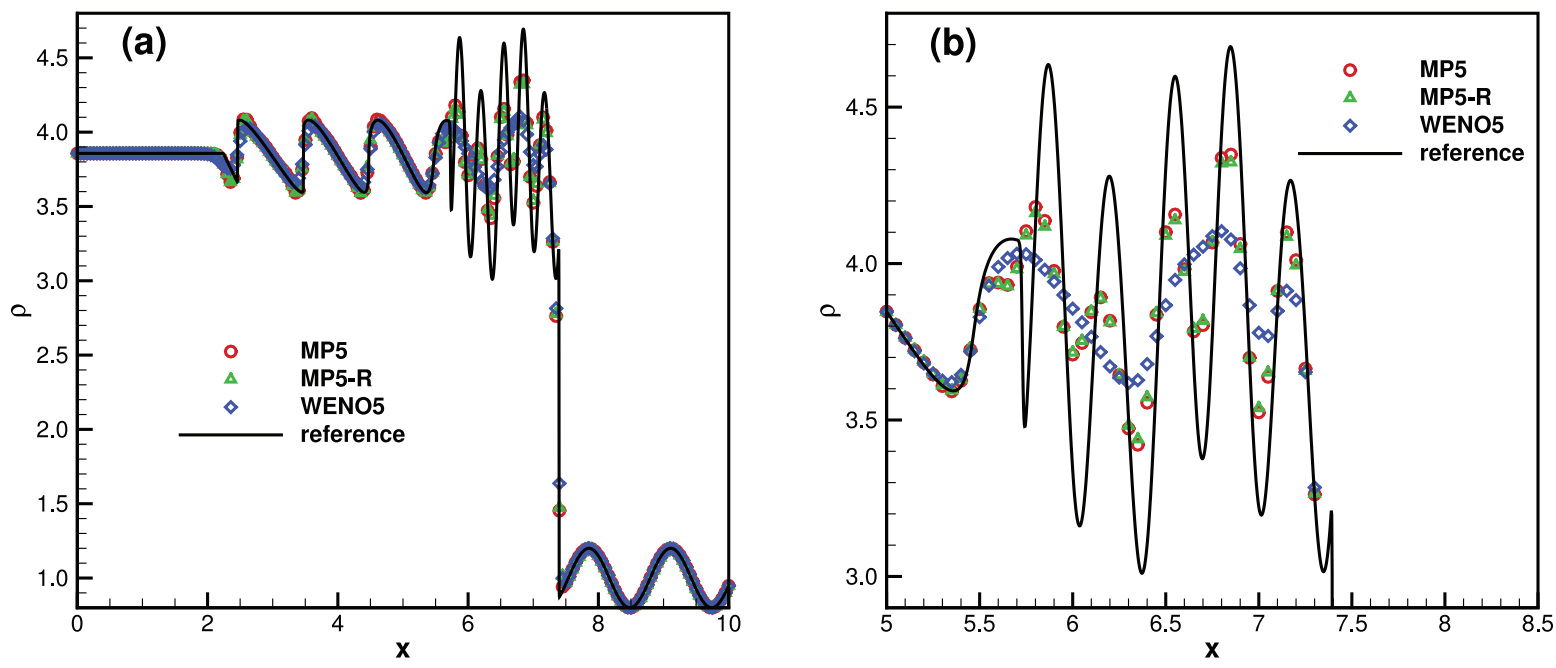

Fig. 4. Shock-density wave interaction on a 201 points grid: (a) density profiles obtained by MP5-R, MP5 and WENO5; (b) enlarged portion of (a).
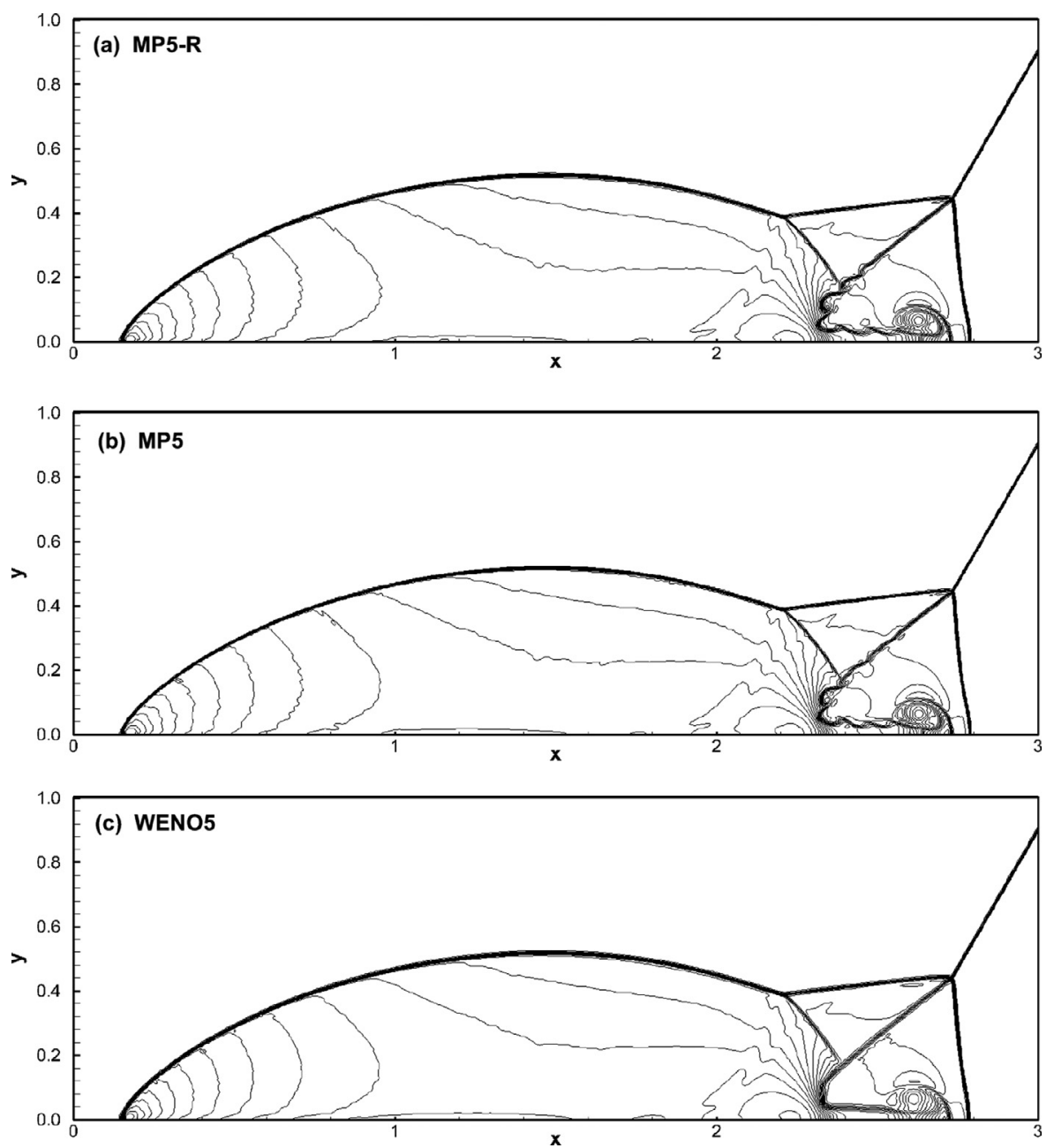

Fig. 5. Double-Mach reflection of a Mach 10 shock wave at $t=0.2$ : 30 density contours from 1.88783 to 20.9144 obtained by (a) MP5-R; (b) MP5; (c) WENO5.

\subsubsection{Double Mach reflection problem}

The computational domain is $[0,4] \times[0,1]$, and the reflecting wall lies at the bottom of the computational domain for $1 / 6$ $\leq x \leq 4$. Initially a right-moving Mach 10 shock is positioned at $(x, y)=(1 / 6,0)$ and makes a $60^{\circ}$ angle with the $x$-axis. For the bottom boundary, the exact post-shock conation is imposed for the part from $x=0$ to $x=1 / 6$, and a reflective boundary condition is used for the rest. At the top boundary, the flow values are set to describe the exact motion of the Mach 10 shock. Inflow and outflow boundary conditions are used for the left and right 

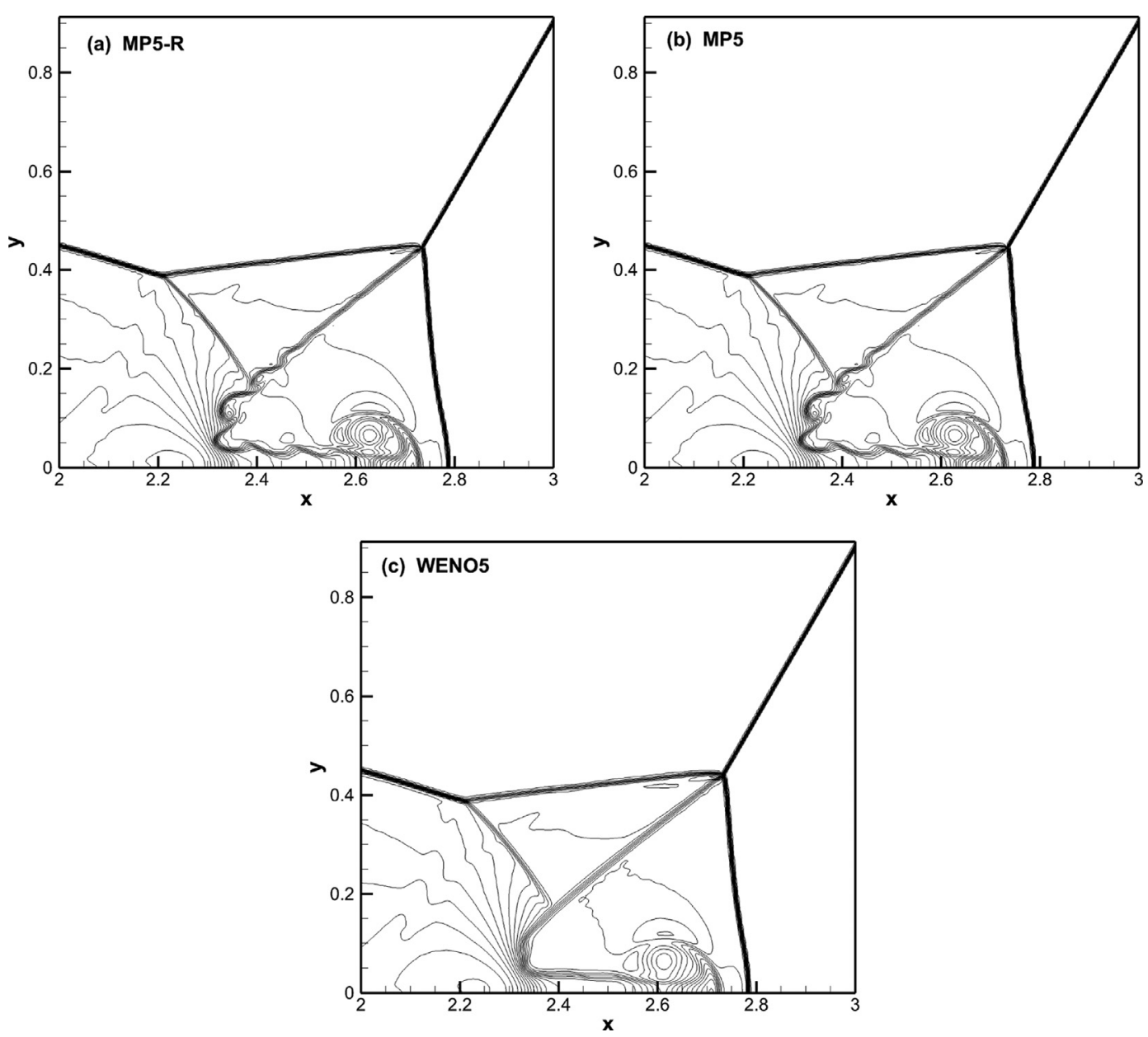

Fig. 6. Close-up view of the "blow-up" region of: (a) Fig. 5(a); (b) Fig. 5(b); (c) Fig. 5(c).

boundaries. The unshocked fluid has a density of 1.4 and a pressure of 1 . The problem was run till $t=0.2$, and the results for [0, $3] \times[0,1]$ are displayed.

Fig. 5 shows the density contours of the solution on a $961 \times$ 241 grid. Good agreement with the results at the same resolution in Hu et al. [29] is observed. Note that while the WENO5 scheme predicts a smeared slip line, the MP5-R scheme resolves much better the wave structures near the second triple point and predicts a strong jet near the wall. As shown in the close-up views of the "blow-up" region (Fig. 6), the MP5-R scheme does show a high resolution property.

From Figs. 5 and 6, we can also see that the resolution property of the MP5-R scheme is essentially as high as that of MP5 scheme, while the MP5-R scheme will be shown to have a high robustness.

\subsection{Robustness tests}

In this section, we consider several challenging tests to show the high robustness of the MP5-R scheme. Note that the original MP5 scheme cannot calculate these problems with the same codes due to the appearance of negative pressure.

5.3.1. One-dimensional problems involving very strong discontinuities

We show that the MP5-R scheme passes two one-dimensional test problems involving very strong discontinuities: the two-blastwave interaction problem, and the LeBlanc problem. The latter is an extreme shock-tube problem. For the first problem, the initial condition is

$$
(\rho, u, p)= \begin{cases}(1,0,1000), & \text { if } 0 \leq x \leq 0.1 \\ (1,0,0.01), & \text { if } 0.1 \leq x \leq 0.9 \\ (1,0,100), & \text { if } 0.9 \leq x \leq 1\end{cases}
$$

$N=401$ is used, and the final time is $t=0.038$. Reflective boundary conditions are applied at both $x=0$ and $x=1$. The "reference" solution is a high-resolution numerical solution on 4001 grid points calculated by the WENO5 scheme. For the second problem, the initial condition is

$(\rho, u, p)= \begin{cases}\left(1,0, \frac{2}{3} \times 10^{-1}\right), & \text { if } 0 \leq x \leq 3 \\ \left(10^{-3}, 0, \frac{2}{3} \times 10^{-10}\right), & \text { if } 3 \leq x \leq 9\end{cases}$

the ratio of specific heats $\gamma=5 / 3$, two grids of $\Delta x=9 / 800$ and $\Delta x=9 / 3200$ are used respectively, and the final time is $t=6$.

Fig. 7 gives the computed pressure, density, and velocity distributions. A good agreement with the exact solution is obtained for the two-blast-wave interaction problem. However, due to the high density ratio as well as a high pressure ratio in the LeBlanc problem, an inaccurate numerical result (especially in the shock location), even with a very fine grid, is obtained. This finding, in accordance with the report [30], but beyond the scope of preset paper, reveals that [30] the Eulerian shock-capturing methods work very inefficiently when applied to problems with an initial high density ratio as well as a high pressure ratio, and may give inaccurate numerical results even over a very fine mesh. 

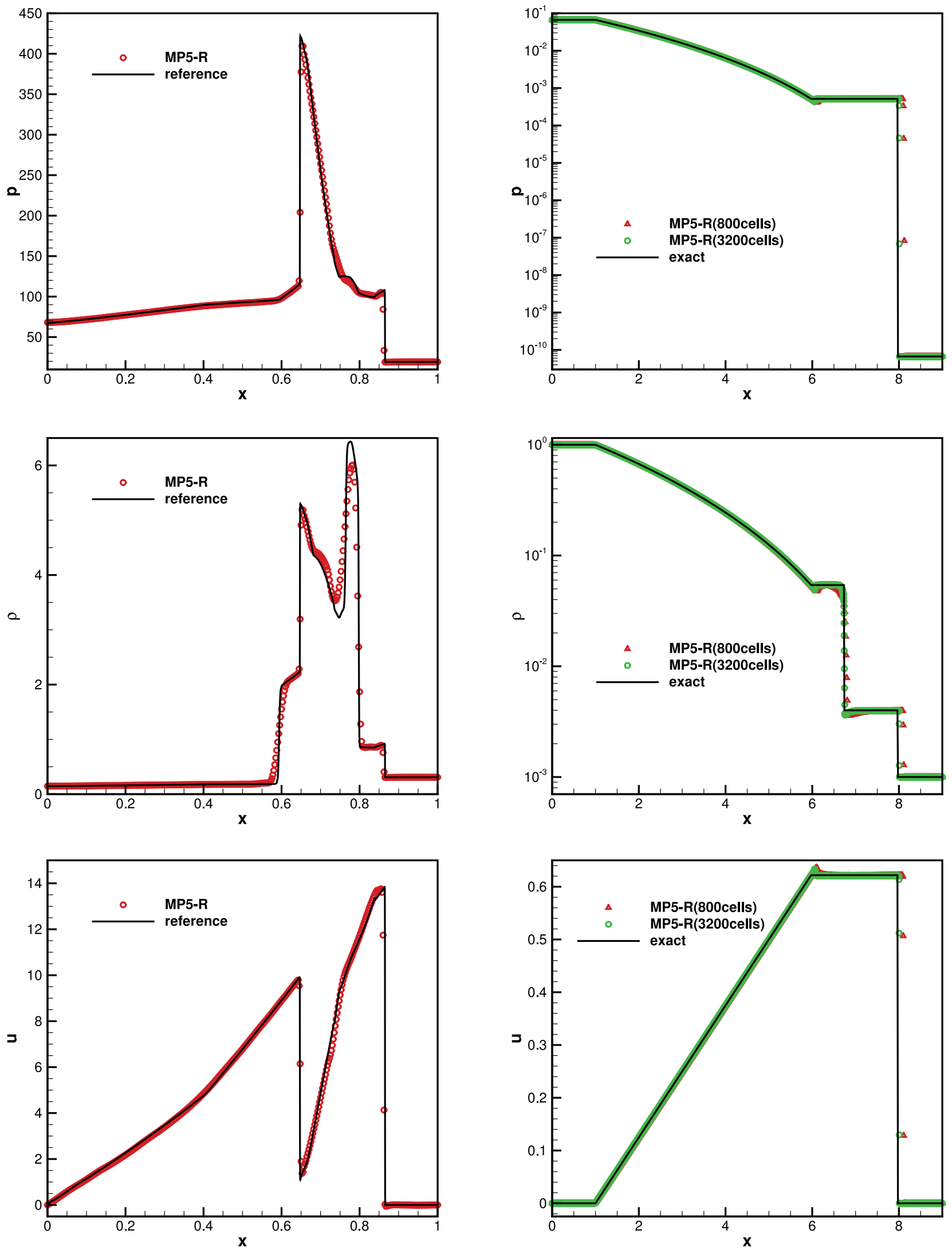

Fig. 7. One-dimensional problems involving very strong discontinuities: (left) two-blast-wave problem; (right) LeBlanc shock-tube problem. 

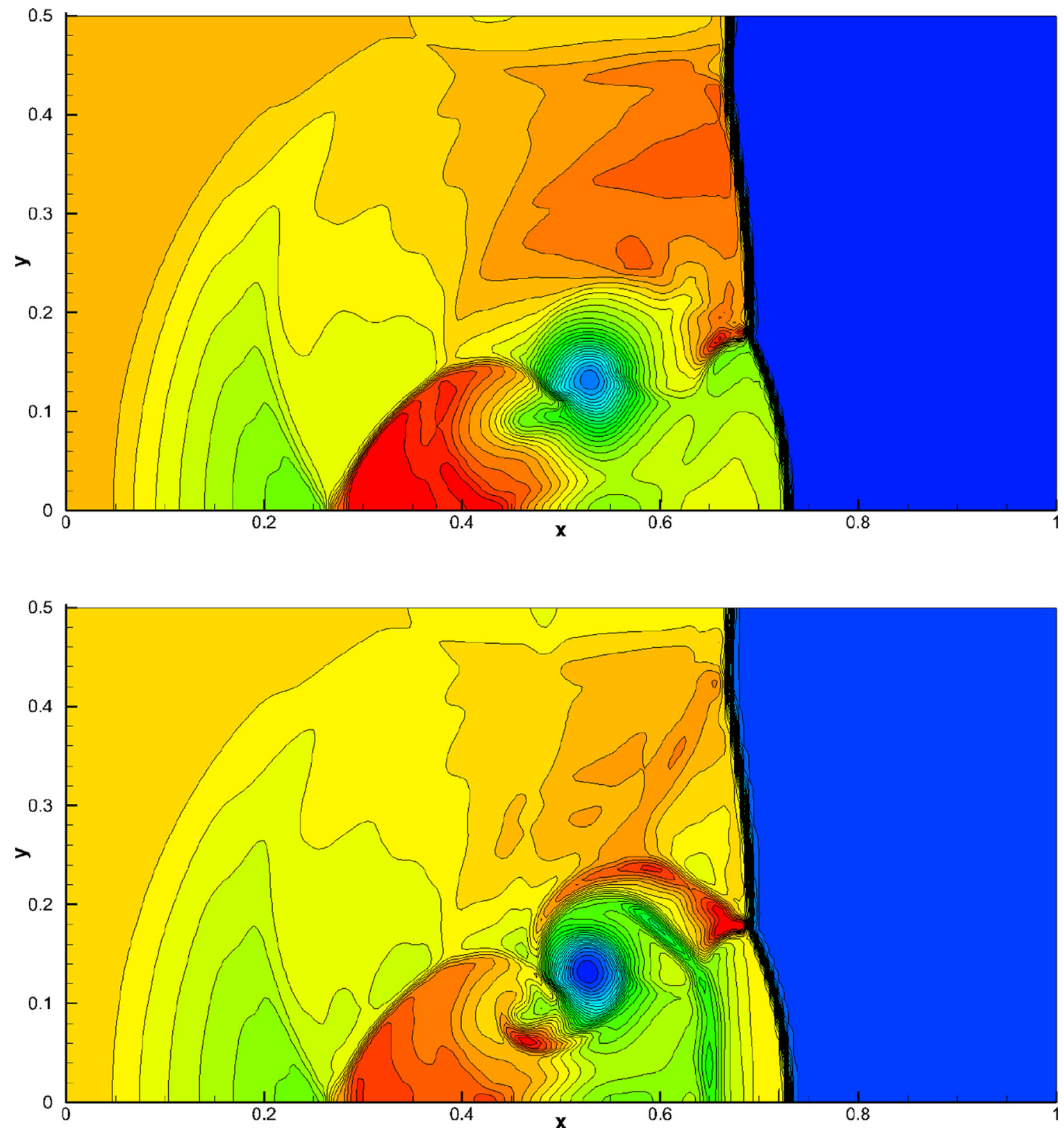

Fig. 8. Shock-bubble interaction problem at $t=0.075$ : (upper) 33 pressure contours from 0.9 to 200 ; (lower) 33 density contours from 0.6 to 7.1 .

\subsubsection{Shock-bubble interaction problem}

For this problem, a very strong shock wave (Mach 12) in air impacts on a cylindrical helium bubble. Air and helium are treated as the same ideal gas fluid for simplicity. Numerical computations for this problem can be found in Bagabir and Drikakis [31]. For this problem, the initial conditions are

$(\rho, u, v, p)=\left\{\begin{array}{l}(1,-6,0,1), \\ (5.799,5.750,0,167.833), \\ (0.138,-6,0,1),\end{array}\right.$

pre-shocked air post-shocked air helium bubble

and the final time is $t=0.075$. The computational domain for this problem is $[0,0] \times[1,0.5]$. Initially, the shock wave is at $x=0.05$, and the half helium bubble of radius 0.15 is at $(0.25,0)$. Note that a frame velocity $u=-6$ is applied to keep the bubble approximately in the center of the computational domain. Reflective conditions are applied at the lower and upper boundaries, an outflow condition is applied at the right boundary, and an inflow condition is applied to the left boundary with the post-shocked state.
Fig. 8 shows the pressure and density contours of the solution on a $201 \times 101$ grid. A good result is obtained by the MP5-R scheme. The second reflected shock wave and triple-wave configurations are calculated with good resolution. It is confirmed again that the MP5-R scheme achieves a good compromise between resolution and robustness.

\subsubsection{Two-dimensional Sedov problem}

Finally, we consider the challenging two-dimensional Sedov problem, which has been studied in Zhang and Shu [27], to demonstrate the high robustness of the MP5-R scheme. The computation is performed on the domain $[0,0] \times[1.1,1.1]$. For the initial condition, the density is 1 , velocity is zero, total energy is $10^{-12}$ everywhere except that the energy in the lower left corner cell is the constant $\frac{0.244816}{\Delta x \Delta y}$. A uniform grid with grid spacing $\Delta x=\Delta y=\frac{1.1}{320}$ is used, and the final time is $t=1$. A reflective boundary condition is applied at the lower and left 


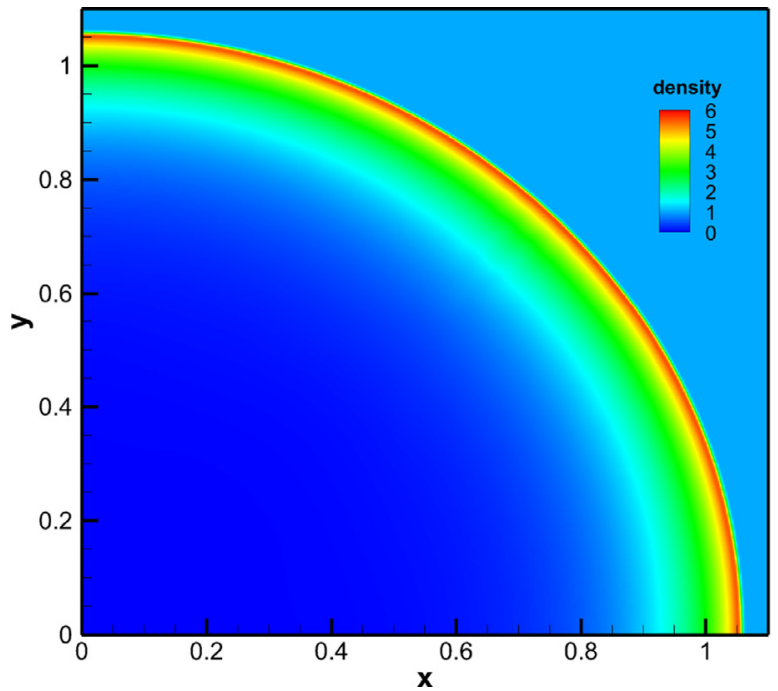

Fig. 9. Two-dimensional Sedov problem. $t=1, \Delta x=\Delta y=\frac{1.1}{320}$ : (left) Color contour of density; (right) Color contour of pressure.

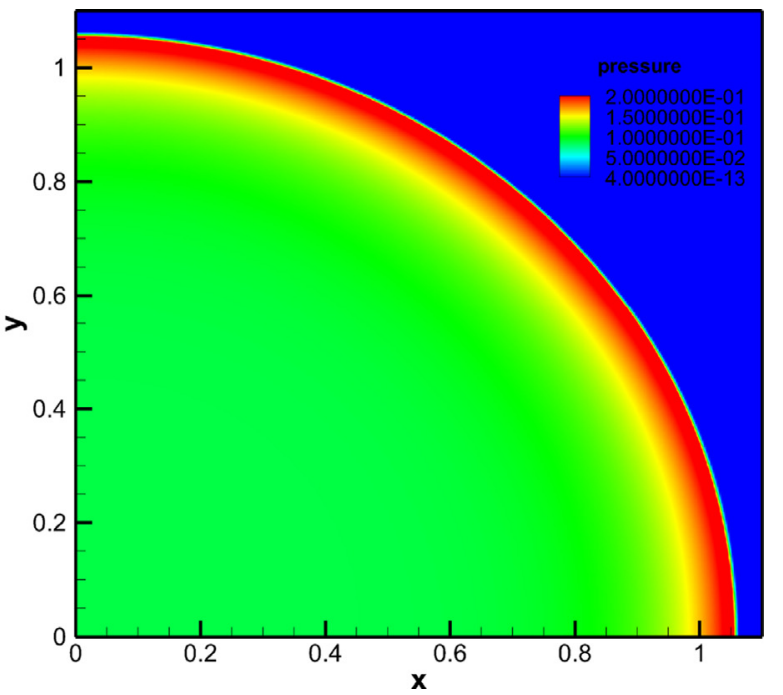

boundaries, and an outflow condition is applied at the right and upper boundaries.

Fig. 9 gives the computed density and pressure contours. This result further confirms our conclusions stated above.

\section{Conclusions}

In this paper, we performed a detailed analysis on the MP scheme, to reveal the potential causes for the weak robustness of the MP scheme. Based on this analysis, a simple modification is proposed, resulting in a new version of the MP scheme. Compared with the original MP scheme, the improved scheme shows not only very high resolution property, but also very high robustness. Therefore, the present scheme is suitable for direct numerical simulation of multi-scale problems with discontinuities, such as the shock wave-turbulent boundary layer interaction problem or Richtmyer-Meshkov instability problem.

\section{Acknowledgment}

This work was supported by NSFC under Grant Nos. 11171037, 11472059, 11372330, 11572052, 11472278, and Key Lab Foundation under Grant No. 9140C690104150C69, and CAEP under Grant No. YZ2015015.

\section{References}

[1] Brouillette M. The Richtmyer -Meshkov instability. Annu Rev Fluid Mech 2002:34:445-68

[2] Li X-L, Fu D-X, Ma Y-W, Liang X. Direct numerical simulation of compressible turbulent flows. Acta Mech Sinica 2010;26:795-806.

[3] van Leer B. Towards the ultimate conservative difference scheme. V. A second order sequel to Godunov's method. J Comput Phys 1979;32:101-36.

[4] Colella P, Woodward PR. The piecewise parabolic method (PPM) for gas-dynamical simulations. J Comput Phys 1984;54:174-201.

[5] Suresh A, Huynh H. Accurate monotonicity-preserving schemes with Runge-Kutta time stepping. J Comput Phys 1997;136:83-99.

[6] Daru V, Tenaud C. High order one-step monotonicity-preserving schemes for unsteady compressible flow calculations. J Comput Phys 2004;193:563-94.

[7] He Z-W, Li X-L, Fu D-X, Ma Y-W. A 5th order monotonicity-preserving upwind compact difference scheme. Sci China Phys Mechani Astron 2011;54:511-22.

[8] Li X-L, Leng Y, He Z-W. Optimized sixth-order monotonicity-preserving scheme by nonlinear spectral analysis. Int I Numer Meth Fluids 2013;73:560-77.

[9] Fang J, Li Z-R, Lu L-P. An optimized low-dissipation monotonicity-preserving scheme for numerical simulations of high-speed turbulent flows. J Sci Comput 2013;56:67-95
[10] Balsara DS, Shu C-W. Monotonicity preserving weighted essentially nonoscillatory schemes with increasingly high order of accuracy. J Comput Phys 2000;160:405-52.

[11] Zanna LD, Zanotti O, Bucciantini N, Londrillo P. ECHO: a Eulerian conservative high-order scheme for general relativistic magnetohydrodynamics and magnetodynamics. Astron Astrophys 2007;473:11-30. doi:10.1051/0004-6361: 20077093.

[12] Falceta-Gonçalves D, Bonnell I, Kowal G, Lépine JRD, Braga CAS. The onset of large-scale turbulence in the interstellar medium of spiral galaxies. Monthly Notices R Astron Soc 2015;446:973-89. doi:10.1093/mnras/stu2127.

[13] Jiang G-S, Shu C-W. Efficient implementation of weighted ENOschemes. J Comput Phys 1996;126:202-28.

[14] Shu C-W, Osher S. Efficient implementation of essentially non-oscillatory shock-capturing schemes. J Comput Phys 1988;77:439-71.

[15] Huynh HT. Accurate monotone cubic interpolation. SIAM J Numer Anal 1993;30:57-100.

[16] Arora M, Roe PL. On the postshock oscillations due to shock capturing schemes in unsteady flows. J Comput Phys 1997;130:25-40.

[17] Bogey C, Bailly C. A family of low dispersive and low dissipative explicit schemes for flow and noise computations. J Comput Phys 2004;194:194-214.

[18] Adams NA, Shariff K. A high-resolution hybrid compact-ENOscheme for shock-turbulence interaction problems. J Comput Phys 1996;127:27-51.

[19] Hu X.-Y., Tritschler V.K., Pirozzoli S., Adams N.A. Dispersion-dissipation condition for finite-difference schemes. arXiv:12045088v22014.

[20] Colonius T, Lele SK. Computational aeroacoustics: progress on nonlinear problems of sound generation. Prog Aerosp Sci 2004;40:345-416.

[21] Sweby P. High resolution schemes using flux limiters for hyperbolic conservation laws. SIAM J Numer Anal 1984;21:995-1011.

[22] Harten A. High resolution schemes for hyperbolic conservation laws. J Comput Phys 1983;49:357-93.

[23] Ren Y-X, Liu M-E, Zhang H-X. A characteristic-wise hybrid compact-WENOscheme for hyperbolic conservation laws. J Comput Phys 2003;192:365-86

[24] van Leer B. Towards the ultimate conservative difference scheme. II. Monotonicity and conservation combined in a second order scheme. J Comput Phys 1974;14:361-70.

[25] Qiu J-X, Shu C-W. On the construction, comparison, and local characteristic decomposition for high-order central WENO schemes. J Comput Phys 2002; 183:187-209.

[26] Shu C-W, Osher S. Efficient implementation of essentially non-oscillatory shock-capturing schemes, II. J Comput Phys 1989;83:32-78.

[27] Zhang X, Shu C-W. Positivity-preserving high order finite difference WENO schemes for compressible Euler equations. J Comput Phys 2012:231:2245-58.

[28] Woodward P, Colella P. The numerical simulation of two-dimensional fluid flow with strong shocks. J Comput Phys 1984;54:115-73.

[29] Hu X-Y, Wang Q, Adams NA. An adaptive central-upwind weighted essentially non-oscillatory scheme. J Comput Phys 2010;229:8952-65.

[30] Tang H, Liu T. A note on the conservative schemes for the Euler equations. J Comput Phys 2006;218:451-9.

[31] Bagabir A, Drikakis D. Mach number effects on shock-bubble interaction. Shock Waves 2001;11:209-18. 